\title{
New algorithms designed for the split common fixed point problem of quasi-pseudocontractions
}

\author{
Li-Jun Zhu' ${ }^{1}$, Yeong-Cheng Liou ${ }^{2,3^{*}}$, Jen-Chih Yao ${ }^{3,4}$ and Yonghong Yao ${ }^{5}$
}

\author{
*Correspondence: \\ simplex_liou@hotmail.com \\ 2Department of Information \\ Management, Cheng Shiu \\ University, Kaohsiung, 833, Taiwan \\ ${ }^{3}$ Center for Fundamental Science, \\ Kaohsiung Medical University, \\ Kaohsiung, 807, Taiwan \\ Full list of author information is \\ available at the end of the article
}

\begin{abstract}
In this paper, we study the split common fixed point problem, which is to find a fixed point of a quasi-pseudocontractive mapping in one space whose image under a linear transformation is a fixed point of anther quasi-pseudocontractive mapping in the image space. We design and analyze a new iterative algorithm for solving this split common fixed point problem. A weak convergence theorem is given.
\end{abstract}

MSC: 49J53; 49M37; 65K10; 90C25

Keywords: split common fixed point; quasi-pseudocontractive mappings; weak convergence

\section{Background and motivation}

Let $C$ and $Q$ be nonempty closed convex subsets of real Hilbert spaces $H_{1}$ and $H_{2}$, respectively. The split feasibility problem is formulated as finding a point $x^{*}$ with the property

$$
x^{*} \in C \text { and } A x^{*} \in Q \text {, }
$$

where $A: H_{1} \rightarrow H_{2}$ is a bounded linear operator. The split feasibility problem in finitedimensional Hilbert spaces was first introduced by Censor and Elfving [1] for modeling inverse problems which arise from phase retrievals and in medical image reconstruction [2].

A special case of the split feasibility problem (1.1) is when $Q=\{b\}$ is singleton and then (1.1) is reduced to the convexly constrained linear inverse problem [3]

$$
x^{*} \in C \text { and } A x^{*}=b \text {, }
$$

which has received considerable attention.

The well-known projected Landweber algorithm [4] is widely used to solve (1.2). This algorithm generates a sequence $\left\{x_{n}\right\}$ in such a way that we have

- initialization: $x_{0}$ selected in $H_{1}$ arbitrarily, and

- iteration:

$$
x_{n+1}=P_{C}\left(x_{n}+\gamma A^{T}\left(b-A x_{n}\right)\right) \text {, }
$$


where $P_{C}$ denotes the nearest point projection from $H_{1}$ onto $C, \gamma>0$ is a parameter such that $0<\gamma<2 /\|A\|^{2}$, and $A^{T}$ is the transpose of $A$.

When the system (1.2) is reduced to the unconstrained linear system

$$
A x^{*}=b,
$$

then the projected Landweber algorithm [4] is turned to the Landweber algorithm:

$$
x_{n+1}=x_{n}+\gamma A^{T}\left(b-A x_{n}\right) .
$$

The simultaneous algebraic reconstruction technique is a typical example of the Landweber algorithm (1.5) when the system (1.4) is finite-dimensional.

The first iterative algorithm for solving the split feasibility problem (1.1) in the finitedimensional case is proposed by Censor and Elfving [1] who define a sequence $x_{n}$ by the recursion:

$$
x_{n+1}=A^{-1} P_{Q}\left(P_{A(C)}\left(A x_{n}\right)\right), \quad n \geq 0,
$$

where $C$ and $Q$ are closed convex sets of $\mathbb{R}^{n}$, and $A$ is an $n \times n$ matrix of full rank. Here $A(C)=\left\{y \in \mathbb{R}^{n}: y=A x, x \in C\right\}$ is the image of $C$ under the matrix $A$.

Because of the presence of the inverse $A^{-1}$, the algorithm (1.6) has not become popular. A more popular algorithm that solves the split feasibility problem (1.1) is the so-called CQ algorithm introduced by Byrne [2]. This algorithm, which does not involve $A^{-1}$, generates a sequence $\left\{x_{n}\right\}$ as follows:

$$
x_{n+1}=P_{C}\left(x_{n}-\gamma A^{T}\left(I-P_{Q}\right) A x_{n}\right), \quad n \geq 0,
$$

where $0<\gamma<2 /\|A\|^{2}$ and $P_{Q}$ denotes the nearest point projection from $H_{2}$ onto $Q$. Consequently, $\mathrm{Xu}[5]$ extend the above results from the finite-dimensional spaces to the infinitedimensional spaces.

In the case where $C$ and $Q$ in (1.1) are the intersections of finitely many fixed point sets of nonlinear operators, problem (1.1) is called by Censor and Segal [6] the split common fixed point problem. More precisely, the split common fixed point problem requires one to seek an element $x^{*} \in H$ satisfying

$$
x^{*} \in \bigcap_{i=1}^{m} \operatorname{Fix}\left(S_{i}\right) \text { and } A x^{*} \in \bigcap_{j=1}^{n} \operatorname{Fix}\left(T_{j}\right) \text {, }
$$

where $\operatorname{Fix}\left(S_{i}\right)$ and $\operatorname{Fix}\left(T_{j}\right)$ denote the fixed point sets of two classes of nonlinear operators $S_{i}: H_{1} \rightarrow H_{1}$ and $T_{j}: H_{2} \rightarrow H_{2}$. In this situation, Byrne's CQ algorithm does not work because the metric projection onto fixed point sets is generally not easy to calculate. To solve the two-set split common fixed point problem, motivated by the algorithms (1.3) and (1.7), Censor and Segal [6] proposed the following iterative method: For any initial guess $x_{1} \in H_{1}$, define $\left\{x_{n}\right\}$ recursively by

$$
x_{n+1}=U\left(x_{n}-\lambda A^{*}(I-T) A x_{n}\right), \quad n \geq 0,
$$


where $U$ and $T$ are directed operators and $\lambda>0$ is known as the step-size. They proved that if $\lambda \in\left(0, \frac{2}{\|A\|^{2}}\right)$, then (1.9) converges to a split common fixed point $x^{*} \in \Gamma=\{x \in$ $\operatorname{Fix}(U) ; A x \in \operatorname{Fix}(T)\}$. Consequently, Moudafi [7] extended (1.9) to the following relaxed algorithm:

$$
\left\{\begin{array}{l}
u_{n}=x_{n}-\gamma A^{*}(I-T) A x_{n}, \\
x_{n+1}=\left(1-\alpha_{n}\right) u_{n}+\alpha_{n} U\left(u_{n}\right), \quad n \in \mathbb{N},
\end{array}\right.
$$

where $U$ and $T$ are demicontractive operators, $\beta \in(0,1), \gamma \in\left(0, \frac{1-\mu}{\lambda}\right)$ with $\lambda$ being the spectral radius of the operator $A^{*} A$ and $\alpha_{n} \in(0,1)$ is relaxation parameter. We note that the classes of directed and demicontractive operators are important classes since they include the orthogonal projections and the subgradient projectors. For some other related work, please refer to [8-26] and [27].

In the present paper, our main motivation is to extend the classes of directed and demicontractive operators to the class of quasi-pseudocontractions because the class of quasi-pseudocontractions includes the classes of directed and demicontractive operators as special cases. Interest in pseudocontractive mappings stems mainly from their firm connection with the class of monotone operators. We present a unified framework for the study of this problem and this class of operators. We propose an iterative algorithm and study its convergence.

\section{Notations and lemmas}

Let $H$ be a real Hilbert space with inner product $\langle\cdot, \cdot\rangle$ and norm $\|\cdot\|$, respectively. Let $C$ be a nonempty closed convex subset of $H$.

Recall that a mapping $T: C \rightarrow C$ is called

- nonexpansive if $\|T x-T y\| \leq\|x-y\|$ for all $x, y \in C$;

- quasi-nonexpansive if $\left\|T x-x^{*}\right\| \leq\left\|x-x^{*}\right\|$ for all $x \in C$ and $x^{*} \in \operatorname{Fix}(T)$;

- firmly nonexpansive if $\|T x-T y\|^{2} \leq\|x-y\|^{2}-\|(I-T) x-(I-T) y\|^{2}$ for all $x, y \in C$;

- firmly quasi-nonexpansive if $\left\|T x-x^{*}\right\|^{2} \leq\left\|x-x^{*}\right\|^{2}-\|T x-x\|^{2}$ for all $x \in C$ and $x^{*} \in \operatorname{Fix}(T)$;

- strictly pseudocontractive if $\|T x-T y\|^{2} \leq\|x-y\|^{2}+k\|(I-T) x-(I-T) y\|^{2}$ for all $x, y \in C$, where $k \in[0,1)$;

- directed if $\left\langle T x-x^{*}, T x-x\right\rangle \leq 0$ for all $x \in C$ and $x^{*} \in \operatorname{Fix}(T)$;

- demicontractive if $\left\|T x-x^{*}\right\|^{2} \leq\left\|x-x^{*}\right\|^{2}+k\|T x-x\|^{2}$ for all $x \in C$ and $x^{*} \in \operatorname{Fix}(T)$, where $k \in[0,1)$.

The concept of directed operators was introduced by Bauschke and Combettes [28] who proved that $T: C \rightarrow C$ is directed if and only if

$$
\left\|T x-x^{*}\right\|^{2} \leq\left\|x-x^{*}\right\|^{2}-\|T x-x\|^{2}
$$

for all $x \in C$ and $x^{*} \in \operatorname{Fix}(T)$. It can be seen easily that the class of directed operators coincides with that of firmly quasi-nonexpansive mappings.

From the above definitions, we note that the class of demicontractive operators contains important operators such as the directed operators, the quasi-nonexpansive operators and the strictly pseudocontractive mappings with fixed points. Such a class of operators is 
fundamental because they include many types of nonlinear operators arising in applied mathematics and optimization; see for example [29] and references therein.

Recall also that a mapping $T: C \rightarrow C$ is called pseudocontractive if

$$
\langle T x-T y, x-y\rangle \leq\|x-y\|^{2}
$$

for all $x, y \in C$. It is well known that $T$ is pseudocontractive if and only if

$$
\|T x-T y\|^{2} \leq\|x-y\|^{2}+\|(I-T) x-(I-T) y\|^{2}
$$

for all $x, y \in C$ and $T: C \rightarrow C$ is said to be quasi-pseudocontractive if

$$
\left\|T x-x^{*}\right\|^{2} \leq\left\|x-x^{*}\right\|^{2}+\|T x-x\|^{2}
$$

for all $x \in C$ and $x^{*} \in \operatorname{Fix}(T)$.

It is obvious that the class of quasi-pseudocontractive mappings includes the class of demicontractive mappings.

A mapping $T: C \rightarrow C$ is called $L$-Lipschitzian if there exists $L>0$ such that

$$
\|T x-T y\| \leq L\|x-y\|
$$

for all $x, y \in C$.

Usually, the convergence of fixed point algorithms requires some additional smoothness properties of the mapping $T$ such as demiclosedness.

Recall that a mapping $T$ is said to be demiclosed if, for any sequence $\left\{x_{n}\right\}$ which weakly converges to $\tilde{x}$, and if the sequence $\left\{T\left(x_{n}\right)\right\}$ strongly converges to $z$, we have $T(\tilde{x})=z$.

Observe also that the nonexpansive operators are both quasi-nonexpansive and strictly pseudocontractive maps and are well known for being demiclosed. For the pseudocontractions, the following demiclosedness principle is well known.

Lemma 2.1 ([30]) Let $H$ be a real Hilbert space, $C$ a closed convex subset of $H$. Let $U$ : $C \rightarrow C$ be a continuous pseudocontractive mapping. Then

(i) $\operatorname{Fix}(U)$ is a closed convex subset of $C$,

(ii) $(I-U)$ is demiclosed at zero.

In the next section, we will need to impose the demiclosedness to the quasi-pseudocontractions.

It is well known that in a real Hilbert space $H$, the following equality holds:

$$
\|t x+(1-t) y\|^{2}=t\|x\|^{2}+(1-t)\|y\|^{2}-t(1-t)\|x-y\|^{2}
$$

for all $x, y \in H$ and $t \in[0,1]$.

Lemma 2.2 ([28]) Let $H$ be a Hilbert space and let $\left\{u_{n}\right\}$ be a sequence in $H$ such that there exists a nonempty set $\Omega \subset H$ satisfying the following:

(i) for every $u \in \Omega, \lim _{n}\left\|u_{n}-u\right\|$ exists,

(ii) any weak-cluster point of the sequence $\left\{u_{n}\right\}$ belongs in $\Omega$.

Then there exists $x^{\dagger} \in \Omega$ such that $\left\{u_{n}\right\}$ weakly converges to $x^{\dagger}$. 
In the sequel we shall use the following notations:

1. $\omega_{w}\left(u_{n}\right)=\left\{x: \exists u_{n_{j}} \rightarrow x\right.$ weakly $\}$ denote the weak $\omega$-limit set of $\left\{u_{n}\right\}$;

2. $u_{n} \rightarrow x$ stands for the weak convergence of $\left\{u_{n}\right\}$ to $x$;

3. $u_{n} \rightarrow x$ stands for the strong convergence of $\left\{u_{n}\right\}$ to $x$.

\section{Main results}

In this section, we will focus our attention on the following general two-operator split common fixed point problem:

$$
\text { find } x^{*} \in C \text { such that } A x^{*} \in Q \text {, }
$$

where $A: H_{1} \rightarrow H_{2}$ is a bounded linear operator, $U: H_{1} \rightarrow H_{1}$ is a quasi-pseudocontractive mapping and $\mathrm{T}: \mathrm{H}_{2} \rightarrow \mathrm{H}_{2}$ is a quasi-pseudocontractive mapping with nonempty fixed point sets $\operatorname{Fix}(U)=C$ and $\operatorname{Fix}(T)=Q$, and we denote the solution set of the two-operator split common fixed point problem by

$$
\Gamma=\{x \in C ; A x \in Q\} .
$$

Algorithm 3.1 For $u_{0} \in H_{1}$, define a sequence $\left\{u_{n}\right\}$ as follows:

$$
\left\{\begin{array}{l}
x_{n}=u_{n}+\gamma \nu A^{*}[\eta I+(1-\eta) T((1-\beta) I+\beta T)-I] A u_{n}, \\
y_{n}=\left(1-\xi_{n}\right) x_{n}+\xi_{n} U x_{n}, \\
u_{n+1}=\left[1-\left(1-\delta_{n}\right) \alpha_{n}\right] x_{n}+\left(1-\delta_{n}\right) \alpha_{n} U y_{n}
\end{array}\right.
$$

for all $n \in \mathbb{N}$, where $\gamma, v, \eta$, and $\beta$ are four constants, $\left\{\alpha_{n}\right\},\left\{\delta_{n}\right\}$, and $\left\{\xi_{n}\right\}$ are three sequences in $[0,1]$.

Now, we demonstrate the convergence analysis of the algorithm (3.1).

Theorem 3.2 Let $H_{1}$ and $H_{2}$ be two real Hilbert spaces. Let $A: H_{1} \rightarrow H_{2}$ be a bounded linear operator. Let $U: H_{1} \rightarrow H_{1}$ and $T: H_{2} \rightarrow H_{2}$ be two L-Lipschitzian quasi-pseudocontractions with nonempty $\operatorname{Fix}(U)=C$ and $\operatorname{Fix}(T)=Q$. Assume $T-I$ and $U-I$ are demiclosed at 0 and $\Gamma \neq \emptyset$. If the parameters $\gamma, v, \eta, \beta,\left\{\alpha_{n}\right\},\left\{\delta_{n}\right\}$, and $\left\{\xi_{n}\right\}$ satisfy the following control conditions:

$\left(C_{1}\right): 0<v<1$ and $0<\gamma<\frac{1}{\lambda v}$, where $\lambda$ is the spectral radius of the operator $A^{*} A$;

$\left(\mathrm{C}_{2}\right): 0<\liminf _{n \rightarrow \infty} \alpha_{n} \leq \limsup _{n \rightarrow \infty} \alpha_{n}<1$;

$\left(C_{3}\right): 0<1-\eta \leq \beta<\frac{1}{\sqrt{1+L^{2}}+1}$ and $0<a \leq 1-\delta_{n} \leq \xi_{n}<\frac{1}{\sqrt{1+L^{2}}+1}$ for all $n \in \mathbb{N}$.

Then the sequence $\left\{u_{n}\right\}$ generated by algorithm (3.2) weakly converges to a split common fixed point $\mu \in \Gamma$.

Remark 3.3 Without loss of generality, we may assume that the Lipschitz constant $L>1$. It is obvious that $\beta<\frac{1}{\sqrt{1+L^{2}}+1}<\frac{1}{L}$ for all $n \geq 1$.

Since $\xi_{n}<\frac{1}{\sqrt{1+L^{2}}+1}$, we have

$$
1-2 \xi_{n}-\xi_{n}^{2} L^{2}>0
$$

for all $n \in \mathbb{N}$. 
Proposition 3.4 Let the mapping $T: H_{2} \rightarrow H_{2}$ be L-Lipschitzian with $L>1$. Then

$$
\operatorname{Fix}(T)=\operatorname{Fix}(T((1-\beta) I+\beta T))
$$

for all $\beta \in\left(0, \frac{1}{L}\right)$.

Proof As a matter of fact, $\operatorname{Fix}(T) \subset \operatorname{Fix}(T((1-\beta) I+\beta T))$ is obvious.

Next, we show that $\operatorname{Fix}(T((1-\beta) I+\beta T)) \subset \operatorname{Fix}(T)$.

Take any $x^{*} \in \operatorname{Fix}(T((1-\beta) I+\beta T))$. We have $T((1-\beta) I+\beta T) x^{*}=x^{*}$. Set $S=(1-\beta) I+\beta T$. We have TS $x^{*}=x^{*}$. Write $S x^{*}=y^{*}$. Then $T y^{*}=x^{*}$. Now we show $x^{*}=y^{*}$. In fact,

$$
\begin{aligned}
\left\|x^{*}-y^{*}\right\| & =\left\|T y^{*}-S x^{*}\right\| \\
& =\left\|T y^{*}-(1-\beta) x^{*}-\beta T x^{*}\right\| \\
& =\beta\left\|T y^{*}-T x^{*}\right\| \\
& \leq \beta L\left\|y^{*}-x^{*}\right\| .
\end{aligned}
$$

Since $\beta<\frac{1}{L}$, we deduce $y^{*}=x^{*} \in \operatorname{Fix}(S)=\operatorname{Fix}(T)$. Thus, $x^{*} \in \operatorname{Fix}(T)$. Hence, $\operatorname{Fix}(T((1-$ $\beta) I+\beta T)) \subset \operatorname{Fix}(T)$. Therefore, $\operatorname{Fix}(T((1-\beta) I+\beta T))=\operatorname{Fix}(T)$.

\section{Proposition 3.5}

$$
\left\|\eta x+(1-\eta) T((1-\beta) I+\beta T) x-x^{*}\right\| \leq\left\|x-x^{*}\right\|
$$

for all $x \in H_{2}$ and all $x \in \operatorname{Fix}(T)$.

Proof Since $x^{*} \in \operatorname{Fix}(T)$, we have from (2.1)

$$
\begin{aligned}
\left\|T((1-\beta) I+\beta T) x-x^{*}\right\|^{2} \leq & \left\|(1-\beta)\left(x-x^{*}\right)+\beta\left(T x-x^{*}\right)\right\|^{2} \\
& +\|((1-\beta) I+\beta T) x-T((1-\beta) I+\beta T) x\|^{2}
\end{aligned}
$$

and

$$
\left\|T x-x^{*}\right\|^{2} \leq\left\|x-x^{*}\right\|^{2}+\|T x-x\|^{2}
$$

for all $x \in H_{2}$.

By (3.3), (2.2), and (3.4), we obtain

$$
\begin{aligned}
\| & T((1-\beta) I+\beta T) x-x^{*} \|^{2} \\
\leq & \left\|(1-\beta)\left(x-x^{*}\right)+\beta\left(T x-x^{*}\right)\right\|^{2} \\
& \quad+\|((1-\beta) I+\beta T) x-T((1-\beta) I+\beta T) x\|^{2} \\
= & \|(1-\beta)(x-T((1-\beta) I+\beta T) x)+\beta(T x-T((1-\beta) I+\beta T) x)\|^{2} \\
& \quad+\left\|(1-\beta)\left(x-x^{*}\right)+\beta\left(T x-x^{*}\right)\right\|^{2} \\
= & (1-\beta)\|x-T((1-\beta) I+\beta T) x\|^{2}+\beta\|T x-T((1-\beta) I+\beta T) x\|^{2}
\end{aligned}
$$




$$
\begin{aligned}
& -\beta(1-\beta)\|x-T x\|^{2}+(1-\beta)\left\|x-x^{*}\right\|^{2}+\beta\left\|T x-x^{*}\right\|^{2}-\beta(1-\beta)\|x-T x\|^{2} \\
\leq & (1-\beta)\left\|x-x^{*}\right\|^{2}+\beta\left(\left\|x-x^{*}\right\|^{2}+\|x-T x\|^{2}\right) \\
& -2 \beta(1-\beta)\|x-T x\|^{2}+(1-\beta)\|x-T((1-\beta) I+\beta T) x\|^{2} \\
& +\beta\|T x-T((1-\beta) I+\beta T) x\|^{2} .
\end{aligned}
$$

Noting that $T$ is $L$-Lipschitzian and $x-((1-\beta) I+\beta T) x=\beta(x-T x)$, we have

$$
\begin{aligned}
\| & T((1-\beta) I+\beta T) x-x^{*} \|^{2} \\
\leq & (1-\beta)\left\|x-x^{*}\right\|^{2}+\beta\left(\left\|x-x^{*}\right\|^{2}+\|x-T x\|^{2}\right) \\
& \quad-2 \beta(1-\beta)\|x-T x\|^{2}+(1-\beta)\|x-T((1-\beta) I+\beta T) x\|^{2}+\beta^{3} L^{2}\|x-T x\|^{2} \\
& =\left\|x-x^{*}\right\|^{2}+(1-\beta)\|x-T((1-\beta) I+\beta T) x\|^{2}-\beta\left(1-2 \beta-\beta^{2} L^{2}\right)\|x-T x\|^{2} .
\end{aligned}
$$

Since $\beta<\frac{1}{\sqrt{1+L^{2}}+1}$, we have

$$
1-2 \beta-\beta^{2} L^{2}>0
$$

From (3.5), we can deduce

$$
\left\|T((1-\beta) I+\beta T) x-x^{*}\right\|^{2} \leq\left\|x-x^{*}\right\|^{2}+(1-\beta)\|x-T((1-\beta) I+\beta T) x\|^{2}
$$

for all $x \in H_{2}$ and $x^{*} \in \operatorname{Fix}(T)$.

Hence,

$$
\begin{aligned}
\| \eta x+ & (1-\eta) T((1-\beta) I+\beta T) x-x^{*} \|^{2} \\
\leq & \left\|\eta\left(x-x^{*}\right)+(1-\eta)\left(T((1-\beta) I+\beta T) x-x^{*}\right)\right\|^{2} \\
= & \eta\left\|x-x^{*}\right\|^{2}+(1-\eta)\left\|T((1-\beta) I+\beta T) x-x^{*}\right\|^{2} \\
& \quad-\eta(1-\eta)\|T((1-\beta) I+\beta T) x-x\|^{2} \\
\leq & \eta\left\|x-x^{*}\right\|^{2}+(1-\eta)\left[\left\|x-x^{*}\right\|^{2}+(1-\beta)\|x-T((1-\beta) I+\beta T) x\|^{2}\right] \\
& \quad-\eta(1-\eta)\|T((1-\beta) I+\beta T) x-x\|^{2} \\
= & \left\|x-x^{*}\right\|^{2}+(1-\eta)(1-\beta-\eta)\|T((1-\beta) I+\beta T) x-x\|^{2} .
\end{aligned}
$$

By $\left(\mathrm{C}_{3}\right)$ and (3.7), we deduce

$$
\left\|\eta x+(1-\eta) T((1-\beta) I+\beta T) x-x^{*}\right\| \leq\left\|x-x^{*}\right\| .
$$

Proposition 3.6 Let the mapping $\mathrm{T}: \mathrm{H}_{2} \rightarrow \mathrm{H}_{2}$ be L-Lipschitzian with $L>1$. If $T-I$ is demiclosed at 0 , then $T((1-\beta) I+\beta T)-I$ is also demiclosed at 0 when $\beta \in\left(0, \frac{1}{L}\right)$.

Proof Let the sequence $\left\{x_{n}\right\} \subset H_{2}$ satisfying $x_{n}-\tilde{x}$ and $x_{n}-T((1-\beta) I+\beta T) x_{n} \rightarrow 0$. Next, we will show that $\tilde{x} \in \operatorname{Fix}(T((1-\beta) I+\beta T))$. 
From Proposition 3.4, we only need to prove that $\tilde{x} \in \operatorname{Fix}(T)$. As a matter of fact, since $T$ is $L$-Lipschitzian, we have

$$
\begin{aligned}
\left\|x_{n}-T x_{n}\right\| & \leq\left\|x_{n}-T((1-\beta) I+\beta T) x_{n}\right\|+\left\|T((1-\beta) I+\beta T) x_{n}-T x_{n}\right\| \\
& \leq\left\|x_{n}-T((1-\beta) I+\beta T) x_{n}\right\|+\beta L\left\|x_{n}-T x_{n}\right\| .
\end{aligned}
$$

It follows that

$$
\left\|x_{n}-T x_{n}\right\| \leq \frac{1}{1-\beta L}\left\|x_{n}-T((1-\beta) I+\beta T) x_{n}\right\| .
$$

Hence,

$$
\lim _{n \rightarrow \infty}\left\|x_{n}-T x_{n}\right\|=0
$$

Applying the demiclosedness of $T$, we immediately deduce $\tilde{x} \in \operatorname{Fix}(T)$.

Next, we prove Theorem 3.2.

Proof Let $x^{*} \in \Gamma$. Then we get $x^{*} \in \operatorname{Fix}(U)$ and $A x^{*} \in \operatorname{Fix}(T)$. From (2.2) and (3.2), we have

$$
\begin{aligned}
\left\|u_{n+1}-x^{*}\right\|^{2}= & \left\|\left[1-\left(1-\delta_{n}\right) \alpha_{n}\right] x_{n}+\left(1-\delta_{n}\right) \alpha_{n} U y_{n}-x^{*}\right\|^{2} \\
= & \left\|\left(1-\alpha_{n}\right)\left(x_{n}-x^{*}\right)+\alpha_{n}\left[\delta_{n} x_{n}+\left(1-\delta_{n}\right) U y_{n}-x^{*}\right]\right\|^{2} \\
= & \left(1-\alpha_{n}\right)\left\|x_{n}-x^{*}\right\|^{2}+\alpha_{n}\left\|\delta_{n} x_{n}+\left(1-\delta_{n}\right) U y_{n}-x^{*}\right\|^{2} \\
& -\alpha_{n}\left(1-\alpha_{n}\right)\left\|\delta_{n} x_{n}+\left(1-\delta_{n}\right) U y_{n}-x_{n}\right\|^{2} \\
= & \alpha_{n}\left[\delta_{n}\left\|x_{n}-x^{*}\right\|^{2}+\left(1-\delta_{n}\right)\left\|U y_{n}-x^{*}\right\|^{2}-\delta_{n}\left(1-\delta_{n}\right)\left\|U y_{n}-x_{n}\right\|^{2}\right] \\
& +\left(1-\alpha_{n}\right)\left\|x_{n}-x^{*}\right\|^{2}-\alpha_{n}\left(1-\alpha_{n}\right)\left\|\delta_{n} x_{n}+\left(1-\delta_{n}\right) U y_{n}-x_{n}\right\|^{2} .
\end{aligned}
$$

Since $x^{*} \in \operatorname{Fix}(U)$, we have from $(2.1)$

$$
\left\|U x-x^{*}\right\|^{2} \leq\left\|x-x^{*}\right\|^{2}+\|x-U x\|^{2}
$$

for all $x \in C$.

By a similar argument to that of (3.6), we obtain

$$
\left\|U y_{n}-x^{*}\right\|^{2} \leq\left\|x_{n}-x^{*}\right\|^{2}+\left(1-\xi_{n}\right)\left\|x_{n}-U y_{n}\right\|^{2} .
$$

Substituting (3.10) to (3.8) and noting that $1-\xi_{n} \leq \delta_{n}$, we have

$$
\begin{aligned}
\left\|u_{n+1}-x^{*}\right\|^{2} \leq & \left(1-\alpha_{n}\right)\left\|x_{n}-x^{*}\right\|^{2}+\alpha_{n}\left\{\delta_{n}\left\|x_{n}-x^{*}\right\|^{2}+\left(1-\delta_{n}\right)\left[\left\|x_{n}-x^{*}\right\|^{2}\right.\right. \\
& \left.\left.+\left(1-\xi_{n}\right)\left\|x_{n}-U y_{n}\right\|^{2}\right]-\delta_{n}\left(1-\delta_{n}\right)\left\|U y_{n}-x_{n}\right\|^{2}\right\} \\
& -\alpha_{n}\left(1-\alpha_{n}\right)\left\|\delta_{n} x_{n}+\left(1-\delta_{n}\right) U y_{n}-x_{n}\right\|^{2} \\
= & \left(1-\alpha_{n}\right)\left\|x_{n}-x^{*}\right\|^{2}+\alpha_{n}\left\{\left\|x_{n}-x^{*}\right\|^{2}\right. \\
& \left.+\left(1-\delta_{n}\right)\left(1-\xi_{n}-\delta_{n}\right)\left\|x_{n}-U y_{n}\right\|^{2}\right\}
\end{aligned}
$$




$$
\begin{aligned}
& -\alpha_{n}\left(1-\alpha_{n}\right)\left\|\delta_{n} x_{n}+\left(1-\delta_{n}\right) U y_{n}-x_{n}\right\|^{2} \\
\leq & \left\|x_{n}-x^{*}\right\|^{2}-\alpha_{n}\left(1-\alpha_{n}\right)\left\|\delta_{n} x_{n}+\left(1-\delta_{n}\right) U y_{n}-x_{n}\right\|^{2} .
\end{aligned}
$$

Since $\lambda$ is the spectral radius of the operator $A A^{*}$, we deduce

$$
\begin{aligned}
& \left\langle[\eta I+(1-\eta) T((1-\beta) I+\beta T)-I] A u_{n}, A A^{*}[\eta I+(1-\eta) T((1-\beta) I+\beta T)-I] A u_{n}\right\rangle \\
& \quad \leq \lambda\left\|[\eta I+(1-\eta) T((1-\beta) I+\beta T)-I] A u_{n}\right\|^{2} .
\end{aligned}
$$

This together with (3.2) implies that

$$
\begin{aligned}
\left\|x_{n}-x^{*}\right\|^{2}= & \left\|u_{n}-x^{*}+\gamma \nu A^{*}[\eta I+(1-\eta) T((1-\beta) I+\beta T)-I] A u_{n}\right\|^{2} \\
= & \left\|u_{n}-x^{*}\right\|^{2}+2 \gamma \nu\left\langle A^{*}[\eta I+(1-\eta) T((1-\beta) I+\beta T)-I] A u_{n}, u_{n}-x^{*}\right\rangle \\
& +\gamma^{2} v^{2}\left\|A^{*}[\eta I+(1-\eta) T((1-\beta) I+\beta T)-I] A u_{n}\right\|^{2} \\
= & \left\|u_{n}-x^{*}\right\|^{2}+2 \gamma \nu\left\langle A^{*}[\eta I+(1-\eta) T((1-\beta) I+\beta T)-I] A u_{n}, u_{n}-x^{*}\right\rangle \\
& +\gamma^{2} v^{2}\left\langle[\eta I+(1-\eta) T((1-\beta) I+\beta T)-I] A u_{n},\right. \\
& \left.A A^{*}[\eta I+(1-\eta) T((1-\beta) I+\beta T)-I] A u_{n}\right\rangle \\
\leq & \left\|u_{n}-x^{*}\right\|^{2}+2 \gamma \nu\left\langle A^{*}[\eta I+(1-\eta) T((1-\beta) I+\beta T)-I] A u_{n}, u_{n}-x^{*}\right\rangle \\
& +\gamma^{2} v^{2} \lambda\left\|[\eta I+(1-\eta) T((1-\beta) I+\beta T)-I] A u_{n}\right\|^{2} .
\end{aligned}
$$

By Proposition 3.5 and noting that $A x^{*} \in \operatorname{Fix}(T)$, we have

$$
\left\|[\eta I+(1-\eta) T((1-\beta) I+\beta T)] A u_{n}-A x^{*}\right\| \leq\left\|A u_{n}-A x^{*}\right\| .
$$

At the same time, we have the following equality in Hilbert spaces:

$$
\|x-y\|^{2}=\|x\|^{2}+\|y\|^{2}-2\langle x, y\rangle
$$

In (3.13), picking up $x=[\eta I+(1-\eta) T((1-\beta) I+\beta T)-I] A u_{n}$ and $y=[\eta I+(1-\eta) T((1-$ $\beta) I+\beta T)] A u_{n}-A x^{*}$ we deduce

$$
\begin{aligned}
\left\|A u_{n}-A x^{*}\right\|^{2}= & \|[\eta I+(1-\eta) T((1-\beta) I+\beta T)-I] A u_{n} \\
& -\left\{[\eta I+(1-\eta) T((1-\beta) I+\beta T)] A u_{n}-A x^{*}\right\} \|^{2} \\
= & \left\|[\eta I+(1-\eta) T((1-\beta) I+\beta T)-I] A u_{n}\right\|^{2} \\
& +\left\|[\eta I+(1-\eta) T((1-\beta) I+\beta T)] A u_{n}-A x^{*}\right\|^{2} \\
& -2\left\langle[\eta I+(1-\eta) T((1-\beta) I+\beta T)-I] A u_{n},\right. \\
& {\left.[\eta I+(1-\eta) T((1-\beta) I+\beta T)] A u_{n}-A x^{*}\right\rangle } \\
\leq & \left\|[\eta I+(1-\eta) T((1-\beta) I+\beta T)-I] A u_{n}\right\|^{2}+\left\|A u_{n}-A x^{*}\right\|^{2} \\
& -2\left\langle[\eta I+(1-\eta) T((1-\beta) I+\beta T)-I] A u_{n},\right. \\
& {\left.[\eta I+(1-\eta) T((1-\beta) I+\beta T)] A u_{n}-A x^{*}\right\rangle . }
\end{aligned}
$$


It follows that

$$
\begin{aligned}
& \left\langle[\eta I+(1-\eta) T((1-\beta) I+\beta T)-I] A u_{n},[\eta I+(1-\eta) T((1-\beta) I+\beta T)] A u_{n}-A x^{*}\right\rangle \\
& \quad \leq \frac{1}{2}\left\|[\eta I+(1-\eta) T((1-\beta) I+\beta T)-I] A u_{n}\right\|^{2} .
\end{aligned}
$$

Thus,

$$
\begin{aligned}
\left\langle A^{*}[\eta I+(1-\eta) T((1-\beta) I+\beta T)-I] A u_{n}, u_{n}-x^{*}\right\rangle \\
=\left\langle[\eta I+(1-\eta) T((1-\beta) I+\beta T)-I] A u_{n}, A u_{n}-A x^{*}\right\rangle \\
=\left\langle[\eta I+(1-\eta) T((1-\beta) I+\beta T)-I] A u_{n},\right. \\
\left.\quad[\eta I+(1-\eta) T((1-\beta) I+\beta T)] A u_{n}-A x^{*}\right\rangle \\
\quad+\left\langle[\eta I+(1-\eta) T((1-\beta) I+\beta T)-I] A u_{n},\right. \\
\left.\quad A u_{n}-[\eta I+(1-\eta) T((1-\beta) I+\beta T)] A u_{n}\right\rangle \\
\leq \frac{1}{2}\left\|[\eta I+(1-\eta) T((1-\beta) I+\beta T)-I] A u_{n}\right\|^{2} \\
\quad-\left\|[\eta I+(1-\eta) T((1-\beta) I+\beta T)-I] A u_{n}\right\|^{2} \\
=-\frac{1}{2}\left\|[\eta I+(1-\eta) T((1-\beta) I+\beta T)-I] A u_{n}\right\|^{2} .
\end{aligned}
$$

From (3.11), (3.12), and (3.14), we get

$$
\begin{aligned}
\left\|u_{n+1}-x^{*}\right\|^{2} \leq & \left\|u_{n}-x^{*}\right\|^{2}-\gamma \nu(1-\lambda \gamma \nu)\left\|[\eta I+(1-\eta) T((1-\beta) I+\beta T)-I] A u_{n}\right\|^{2} \\
& -\alpha_{n}\left(1-\alpha_{n}\right)\left\|\delta_{n} x_{n}+\left(1-\delta_{n}\right) U y_{n}-x_{n}\right\|^{2} .
\end{aligned}
$$

We deduce immediately that

$$
\left\|u_{n+1}-x^{*}\right\| \leq\left\|u_{n}-x^{*}\right\| .
$$

Hence, $\lim _{n \rightarrow \infty}\left\|u_{n}-x^{*}\right\|$ exists. This implies that $\left\{u_{n}\right\}$ is bounded. Consequently, we have

$$
\begin{aligned}
0 & \leq \gamma \nu(1-\lambda \gamma \nu)\left\|[\eta I+(1-\eta) T((1-\beta) I+\beta T)-I] A u_{n}\right\|^{2} \\
& \leq\left\|u_{n}-x^{*}\right\|^{2}-\left\|u_{n+1}-x^{*}\right\|^{2} \rightarrow 0
\end{aligned}
$$

Therefore,

$$
\lim _{n \rightarrow \infty}\left\|[\eta I+(1-\eta) T((1-\beta) I+\beta T)-I] A u_{n}\right\|=0
$$

Since $\left\{u_{n}\right\}$ is bounded, $\omega_{w}\left(u_{n}\right) \neq \emptyset$. We can take $\mu \in \omega_{w}\left(u_{n}\right)$, that is, there exists $\left\{u_{n_{j}}\right\}$ such that $\omega-\lim _{j \rightarrow \infty} u_{n_{j}}=\mu$. Since $T-I$ is demiclosed at 0 , by Proposition 3.6, we see that $T((1-\beta) I+\beta T)-I$ is also demiclosed at 0 . Then, from (3.16), we obtain

$$
[\eta I+(1-\eta) T((1-\beta) I+\beta T)-I] A \mu=0 .
$$

Thus, $A \mu \in \operatorname{Fix}(T((1-\beta) I+\beta T))=\operatorname{Fix}(T)$. 
From (3.15), we deduce

$$
\alpha_{n}\left(1-\alpha_{n}\right)\left\|\delta_{n} x_{n}+\left(1-\delta_{n}\right) U y_{n}-x_{n}\right\|^{2} \leq\left\|u_{n}-x^{*}\right\|^{2}-\left\|u_{n+1}-x^{*}\right\|^{2} \rightarrow 0 .
$$

This together with $\left(C_{2}\right)$ implies that

$$
\lim _{n \rightarrow \infty}\left\|\delta_{n} x_{n}+\left(1-\delta_{n}\right) U y_{n}-x_{n}\right\|=\lim _{n \rightarrow \infty}\left(1-\delta_{n}\right)\left\|U y_{n}-x_{n}\right\|=0 .
$$

Noticing that $1-\delta_{n} \geq a$, we get immediately

$$
\lim _{n \rightarrow \infty}\left\|U y_{n}-x_{n}\right\|=0 .
$$

Since $U$ is $L$-Lipschitzian, we have

$$
\begin{aligned}
\left\|U x_{n}-x_{n}\right\| & \leq\left\|U x_{n}-U y_{n}\right\|+\left\|U y_{n}-x_{n}\right\| \\
& \leq L\left\|x_{n}-y_{n}\right\|+\left\|U y_{n}-x_{n}\right\| \\
& =L \xi_{n}\left\|U x_{n}-x_{n}\right\|+\left\|U y_{n}-x_{n}\right\| .
\end{aligned}
$$

It follows that

$$
\left\|U x_{n}-x_{n}\right\| \leq \frac{1}{1-L \xi_{n}}\left\|U y_{n}-x_{n}\right\|
$$

Since $\xi_{n}<\frac{1}{\sqrt{1+L^{2}}+1}<\frac{1}{L}$, we deduce

$$
\lim _{n \rightarrow \infty}\left\|U x_{n}-x_{n}\right\|=0 .
$$

From (3.2) and (3.16), we have $\lim _{n \rightarrow \infty}\left\|x_{n}-u_{n}\right\|=0$. Thus, $\omega-\lim _{j \rightarrow \infty} x_{n_{j}}=\mu$. By the demiclosedness of $U-I$ at 0 and (3.17), we get $\mu \in \operatorname{Fix}(U)$. Hence, $\mu \in \operatorname{Fix}(U)$. Therefore, $\mu \in \Gamma$.

Note that there is no more than one weak-cluster point of $\left\{u_{n}\right\}$. In fact, if we assume there exists another $\left\{u_{n_{k}}\right\}$ such that $\omega-\lim _{k \rightarrow \infty} u_{n_{k}}=\tilde{\mu} \neq \mu$, then we can deduce $\tilde{\mu} \in \operatorname{Fix}(U)$. Now we show $\tilde{\mu}=\mu$. By the Opial property of Hilbert space, we have

$$
\begin{aligned}
\liminf _{k \rightarrow \infty}\left\|u_{n_{k}}-\tilde{\mu}\right\| & <\liminf _{k \rightarrow \infty}\left\|u_{n_{k}}-\mu\right\|=\lim _{n \rightarrow \infty}\left\|u_{n}-\mu\right\| \\
& =\liminf _{j \rightarrow \infty}\left\|u_{n_{j}}-\mu\right\|<\liminf _{j \rightarrow \infty}\left\|u_{n_{j}}-\tilde{\mu}\right\| \\
& =\lim _{n \rightarrow \infty}\left\|u_{n}-\tilde{\mu}\right\| \\
& =\liminf _{k \rightarrow \infty}\left\|u_{n_{k}}-\tilde{\mu}\right\| .
\end{aligned}
$$

This is a contradiction. Hence, the weak convergence of the whole sequence $\left\{u_{n}\right\}$ follows by applying Lemma 2.2 with $\Omega=\Gamma$. This completes the proof.

Remark 3.7 Since the class of quasi-pseudocontractions contains the demicontractive operators, the directed operators, the quasi-nonexpansive operators and the strictly pseudocontractive mappings with fixed points as special cases, our results present a unified framework for the study of this problem and this class of operators. 
Corollary 3.8 Let $H_{1}$ and $H_{2}$ be two real Hilbert spaces. Let $A: H_{1} \rightarrow H_{2}$ be a bounded linear operator. Let $U: H_{1} \rightarrow H_{1}$ and $T: H_{2} \rightarrow H_{2}$ be two L-Lipschitzian demicontractive mappings with nonempty $\operatorname{Fix}(U)=C$ and $\operatorname{Fix}(T)=Q$. Assume $T-I$ and $U-I$ are demiclosed at 0 and $\Gamma \neq \emptyset$. If the parameters $\gamma, v, \eta, \beta,\left\{\alpha_{n}\right\},\left\{\delta_{n}\right\}$ and $\left\{\xi_{n}\right\}$ satisfy the following control conditions:

$\left(C_{1}\right): 0<v<1$ and $0<\gamma<\frac{1}{\lambda v}$, where $\lambda$ is the spectral radius of the operator $A^{*} A$;

$\left(C_{2}\right): 0<\liminf _{n \rightarrow \infty} \alpha_{n} \leq \limsup _{n \rightarrow \infty} \alpha_{n}<1$;

$\left(C_{3}\right): 0<1-\eta \leq \beta<\frac{1}{\sqrt{1+L^{2}}+1}$ and $0<a \leq 1-\delta_{n} \leq \xi_{n}<\frac{1}{\sqrt{1+L^{2}}+1}$ for all $n \in \mathbb{N}$.

Then the sequence $\left\{u_{n}\right\}$ generated by algorithm (3.2) weakly converges to a split common fixed point $\mu \in \Gamma$.

\section{Competing interests}

The authors declare that they have no competing interests.

\section{Authors' contributions}

All authors read and approved the final manuscript.

\section{Author details}

'School of Mathematics and Information Science, Beifang University of Nationalities, Yinchuan, 750021, China.

${ }^{2}$ Department of Information Management, Cheng Shiu University, Kaohsiung, 833, Taiwan. ${ }^{3}$ Center for Fundamental Science, Kaohsiung Medical University, Kaohsiung, 807, Taiwan. ${ }^{4}$ Department of Mathematics, King Abdulaziz University, P.O. Box 80203, Jeddah, 21589, Saudi Arabia. ${ }^{5}$ Department of Mathematics, Tianjin Polytechnic University, Tianjin, 300387 , China.

\section{Acknowledgements}

Li-Jun Zhu was supported in part by NNSF of China (61362033). Yeong-Cheng Liou was supported in part by the Grants NSC 101-2628-E-230-001-MY3 and NSC 103-2923-E-037-001-MY3. Jen-Chih Yao was partially supported by the Grant NSC 103-2923-E-037-001-MY3.

Received: 22 April 2014 Accepted: 15 July 2014 Published: 19 Aug 2014

\section{References}

1. Censor, Y, Elfving, T: A multiprojection algorithm using Bregman projections in a product space. Numer. Algorithms 8 , 221-239 (1994)

2. Byrne, C: Iterative oblique projection onto convex subsets and the split feasibility problem. Inverse Probl. 18, 441-453 (2002)

3. Eicke, B: Iteration methods for convexly constrained ill-posed problems in Hilbert spaces. Numer. Funct. Anal. Optim. 13, 413-429 (1992)

4. Landweber, L: An iterative formula for Fredholm integral equations of the first kind. Am. J. Math. 73, 615-624 (1951)

5. Xu, HK: Iterative methods for the split feasibility problem in infinite-dimensional Hilbert spaces. Inverse Probl. 26, 105018 (2010)

6. Censor, Y, Segal, A: The split common fixed point problem for directed operators. J. Convex Anal. 16, 587-600 (2009)

7. Moudafi, A: A note on the split common fixed-point problem for quasi-nonexpansive operators. Nonlinear Anal. 74, 4083-4087 (2011)

8. Ceng, LC, Ansari, QH, Yao, JC: An extragradient method for split feasibility and fixed point problems. Comput. Math. Appl. 64, 633-642 (2012)

9. Ceng, LC, Ansari, QH, Yao, JC: Relaxed extragradient methods for finding minimum-norm solutions of the split feasibility problem. Nonlinear Anal. 75, 2116-2125 (2012)

10. Ceng, LC, Yao, JC: Relaxed and hybrid viscosity methods for general system of variational inequalities with split feasibility problem constraint. Fixed Point Theory Appl. 2013, 43 (2013)

11. Dang, Y, Gao, Y: The strong convergence of a KM-CQ-like algorithm for a split feasibility problem. Inverse Probl. 27, 015007 (2011)

12. Wang, F, Xu, HK: Approximating curve and strong convergence of the CQ algorithm for the split feasibility problem. J. Inequal. Appl. 2010, Article ID 102085 (2010)

13. Wang, F, Xu, HK: Cyclic algorithms for split feasibility problems in Hilbert spaces. Nonlinear Anal. 74, 4105-4111 (2011)

14. Yang, Q: The relaxed CQ algorithm solving the split feasibility problem. Inverse Probl. 20, 1261-1266 (2004)

15. Yao, Y, Wu, J, Liou, YC: Regularized methods for the split feasibility problem. Abstr. Appl. Anal. 2012, Article ID 140679 (2012)

16. Yu, X, Shahzad, N, Yao, Y: Implicit and explicit algorithms for solving the split feasibility problem. Optim. Lett. 6, 1447-1462 (2012)

17. Yao, Y, Kim, TH, Chebbi, S, Xu, HK: A modified extragradient method for the split feasibility and fixed point problems. J. Nonlinear Convex Anal. 13, 383-396 (2012) 
18. Zhao, J, Yang, Q: Several solution methods for the split feasibility problem. Inverse Probl. 21, 1791-1799 (2005)

19. He, ZH, Du, WS: On hybrid split problem and its nonlinear algorithms. Fixed Point Theory Appl. 2013, $47(2013)$

20. He, ZH, Du, WS: Nonlinear algorithms approach to split common solution problems. Fixed Point Theory Appl. 2012, $130(2012)$

21. Chang, SS, Wang, L, Tang, YK, Yang, L: The split common fixed point problem for total asymptotically strictly pseudocontractive mappings. J. Appl. Math. 2012, Article ID 385638 (2012)

22. Chang, SS, Kim, J, Cho, YJ, Sim, J: Weak and strong convergence theorems of solutions to split feasibility problem for nonspreading type mapping in Hilbert spaces. Fixed Point Theory Appl. 2014, 11 (2014)

23. Cui, H, Su, M, Wang, F: Damped projection method for split common fixed point problems. J. Inequal. Appl. 2013, 123 (2013). doi:10.1186/1029-242X-2013-123

24. Yao, Y, Postolache, M, Liou, YC: Strong convergence of a self-adaptive method for the split feasibility problem. Fixed Point Theory Appl. 2013, 201 (2013)

25. Zhao, J, He, S: Alternating Mann iterative algorithms for the split common fixed-point problem of quasi-nonexpansive mappings. Fixed Point Theory Appl. 2013, 288 (2013)

26. Deepho, J, Kumam, P: Mann's type extragradient for solving split feasibility and fixed point problems of Lipschitz asymptotically quasi-nonexpansive mappings. Fixed Point Theory Appl. 2013, 349 (2013)

27. Ansari, QH, Rehan, A: Split feasibility and fixed point problems. In: Ansari, QH (ed.) Nonlinear Analysis: Approximation Theory, Optimization and Applications, pp. 271-314. Springer, Berlin (2014)

28. Bauschke, HH, Combettes, PL: A weak-to-strong convergence principle for Feje-monotone methods in Hilbert spaces. Math. Oper. Res. 26, 248-264 (2001)

29. Maruster, S, Popirlan, C: On the Mann-type iteration and convex feasibility problem. J. Comput. Appl. Math. 212, 390-396 (2008)

30. Zhou, H: Strong convergence of an explicit iterative algorithm for continuous pseudo-contractions in Banach spaces. Nonlinear Anal. 70, 4039-4046 (2009)

10.1186/1029-242X-2014-304

Cite this article as: Zhu et al.: New algorithms designed for the split common fixed point problem of quasi-pseudocontractions. Journal of Inequalities and Applications 2014, 2014:304

\section{Submit your manuscript to a SpringerOpen ${ }^{\odot}$ journal and benefit from:}

- Convenient online submission

- Rigorous peer review

Immediate publication on acceptance

Open access: articles freely available online

- High visibility within the field

- Retaining the copyright to your article 